\title{
Flow diversion and microvascular plug occlusion for the treatment of a complex unruptured basilar/superior cerebellar artery aneurysm: case report
}

\author{
Jan-Karl Burkhardt, MD, ${ }^{1}$ Howard A. Riina, MD, ${ }^{1}$ Omar Tanweer, MD, ${ }^{1}$ Peyman Shirani, MD, ${ }^{1,2}$ \\ Eytan Raz, MD, ${ }^{1}$ Maksim Shapiro, MD, ${ }^{1}$ and Peter Kim Nelson, MD' ${ }^{1}$
}

\begin{abstract}
${ }^{1}$ The Irene and Bernard Schwartz Neurointerventional Radiology Center, Departments of Neurosurgery and Radiology, New York University School of Medicine, NYU Langone Medical Center, New York, New York; and 2Department of Neurology, University of Cincinnati, Cincinnati, Ohio
\end{abstract}

\begin{abstract}
The authors present the unusual case of a complex unruptured basilar artery terminus (BAT) aneurysm in a 42-year-old symptomatic female patient presenting with symptoms of mass effect. Due to the fusiform incorporation of both the BAT and left superior cerebellar artery (SCA) origin, simple surgical or endovascular treatment options were not feasible in this case. A 2-staged (combined deconstructive/reconstructive) procedure was successfully performed: first occluding the left SCA with a Pipeline embolization device (PED) coupled to a microvascular plug (MVP) in the absence of antiplatelet coverage, followed by reconstruction of the BAT by deploying a second PED from the right SCA into the basilar trunk. Six-month follow-up angiography confirmed uneventful aneurysm occlusion. The patient recovered well from her neurological symptoms. This case report illustrates the successful use of a combined staged deconstructive/reconstructive endovascular approach utilizing 2 endoluminal tools, PED and MVP, to reconstruct the BAT and occlude a complex aneurysm.
\end{abstract}

https://thejns.org/doi/abs/10.3171/2018.1.JNS172465

KEYWORDS flow diverter; complex basilar artery terminus aneurysm; microvascular plug occlusion; vascular disorders

$\mathrm{A}$ NEURYSMS of the basilar artery (BA) that involve the BA terminus (BAT) are among the more challenging lesions for surgical treatment, especially if the aneurysm incorporates branches of the quadrifurcation, is large in size, or is fusiform in shape.$^{3,5,6}$ Endovascular coiling showed better patient outcome than surgical treatment in randomized clinical trials and is today considered the treatment of choice for aneurysms in this location. ${ }^{11}$ However, endovascular coiling alone is not always feasible for complex and/or fusiform aneurysms, even if stent-assisted coiling or balloon-assisted coiling is performed. ${ }^{7}$ When endovascular coiling fails, surgical treatment with bypass surgery and aneurysm trapping is a possible treatment mo- dality for these complex BAT cases, but it requires surgical experience and has a high rate of complications. ${ }^{8}$

Nowadays, with the introduction of minimally porous endoluminal devices (MPEDs) such as the Pipeline (Pipeline embolization device [PED]) or Silk flow diverter, new treatment strategies are available for management of complex cases. ${ }^{1}$ Here we present the rare case of a patient with a symptomatic complex fusiform BAT/left superior cerebellar artery (SCA) aneurysm, treated successfully with staged combination of deconstructive occlusion of the SCA followed by endoluminal reconstruction of the BAT with a PED flow diverter. 

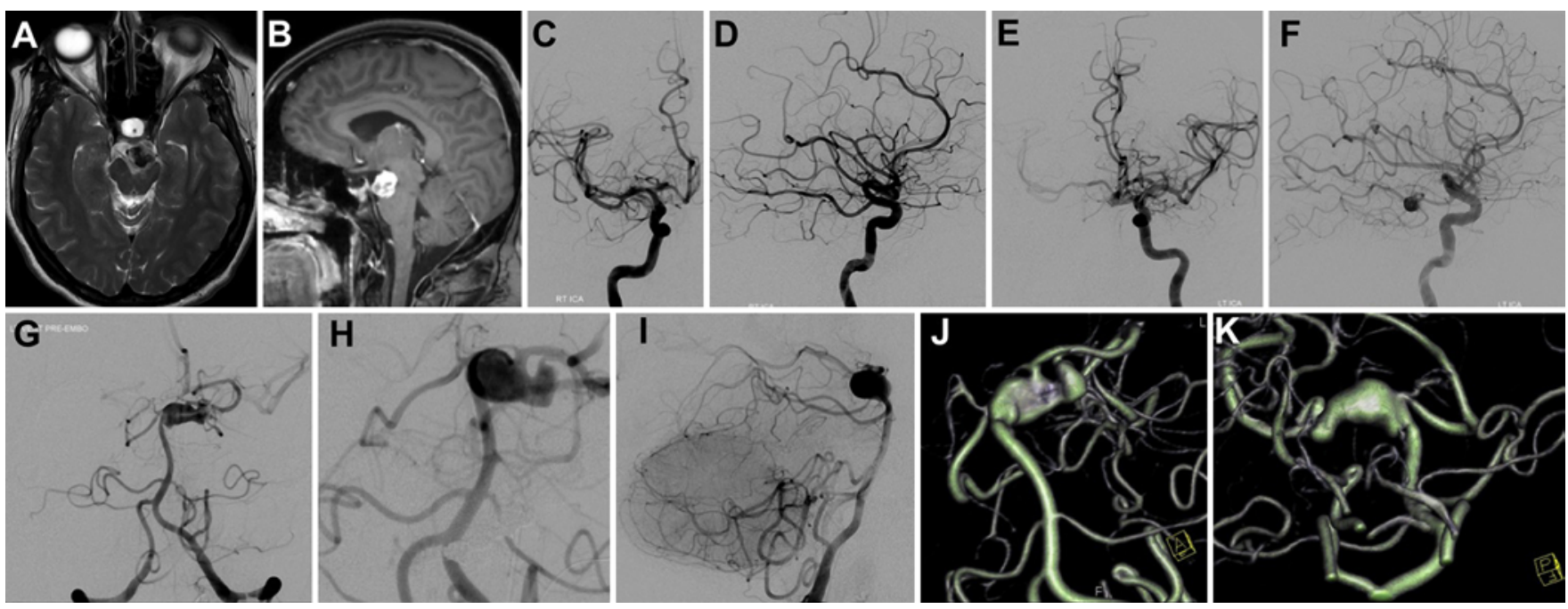

FIG. 1. Initial diagnostic workup. A and B: Axial T2-weighted (A) and sagittal contrast-enhanced T1-weighted (B) MR images showing the BAT aneurysm. C-I: Angiographic images. Anteroposterior and lateral views of the right ICA (C and D), left ICA (E and $F$ ) and left $V A(G-I)$ confirmed the presence of a complex fusiform aneurysm with partial filling through the left PCoA from the left ICA. $\mathrm{J}$ and $\mathrm{K}$ : 3D rotational angiography of the left VA; anterior $(\mathrm{J})$ and posterior $(\mathrm{K})$ views. Figure is available in color online only.

\section{Case Report}

\section{Clinical Presentation and Diagnostic Workup}

This 42-year-old female with a history of chronic headaches presented with right-sided dysmetria, slight right facial weakness, and an unsteady gait. MRI/MRA showed an unruptured BAT aneurysm with brainstem mass effect (Fig. 1A and B). Diagnostic angiography of the right internal carotid artery (ICA) was notable for a fetal-type posterior cerebral artery (PCA) (Fig. 1C and D) coupled with an atretic right $\mathrm{P}_{1}$ segment, and arterial-phase images of the left ICA were notable for a prominent left posterior communicating artery (PCoA). Retrograde opacification of the left $\mathrm{P}_{1}$ segment resulted in transient opacification of the aneurysm and superior cerebellar arteries (SCAs) bilaterally (Fig. 1E and F). Left vertebral artery (VA) angiography delineated a complex fusiform $(10 \times 11 \times 22$ $\mathrm{mm}$ ) BAT/SCA aneurysm (Fig. $1 \mathrm{G}$ and $\mathrm{K}$ ).

Due to the involvement of both the BAT and the SCA, direct surgical clipping or endovascular coiling did not appear indicated in this case. Bypass surgery and aneurysm trapping was discussed with the patient, but in this case the treatment team and the patient decided to proceed with endovascular reconstruction of the BAT. This procedure was planned and performed in 2 stages. First, the plan was to address the funnel-shaped component of the aneurysm involving the proximal left SCA followed by endoluminal reconstruction of the fusiform BAT aneurysm using a PED.

\section{Stage 1-BA Balloon Test Occlusion and Occlusion of the Left SCA Using a Microvascular Plug Deployed Within an Implanted PED}

Following the angiographic evaluation, balloon test occlusion of the BA was performed. For this purpose, a 4-mm-diameter, 15-mm-long Hyperglide balloon microcatheter was coaxially introduced through the indwelling
6-Fr Envoy catheter and positioned within the mid-BA just proximal to the aneurysm. Under live subtraction imaging, the balloon was inflated, and sequential angiograms of the left VA and ICA were obtained for evaluation of collateral reconstitution of the basilar apex (Fig. 2A-C). Of note, there was retrograde reconstitution of the SCAs bilaterally through the left PCoA, reversing flow within the left $\mathrm{P}_{1}$ segment.

The balloon microcatheter was deflated and removed. Under roadmap fluoroscopic guidance, the aneurysm was superselectively catheterized with a 2.8-Fr Marksman microcatheter, and the left lateral fusiform component of the aneurysm (exiting into the left SCA) was deconstructively occluded with a 5-mm-diameter by 10-mm-long PED into which a 5-mm-diameter microvascular plug (MVP) mechanical occlusion device was deployed, proximally arresting antegrade runoff into the left SCA and occluding the SCA-associated fusiform component of the aneurysm. The purpose of combining a PED with an MVP to occlude the SCA componment of the aneurysm was based on the lesion's atypical fusiform morphology. The tapering vascular diameter of this portion of the aneurysmconfluent with exit of a more normative segment of the left SCA-presented an anatomical condition unsuitable for secure solo positioning of an MVP. In this setting, the PED served as an anchoring scaffold (that could be seated into the exiting SCA) to stabilize subsequent placement of the MVP, thereby preventing uncontrolled migration of the MVP more proximally within the aneurysm sac after deployment. Moreover, the use of the PED (without antiplatelet coverage) contributes to the thrombogenicity of the construct, facilitating more immediate occlusion of the ectatic SCA than would have been likely after deployment of an MVP device alone.

Postembolization control angiographies of the left VA 

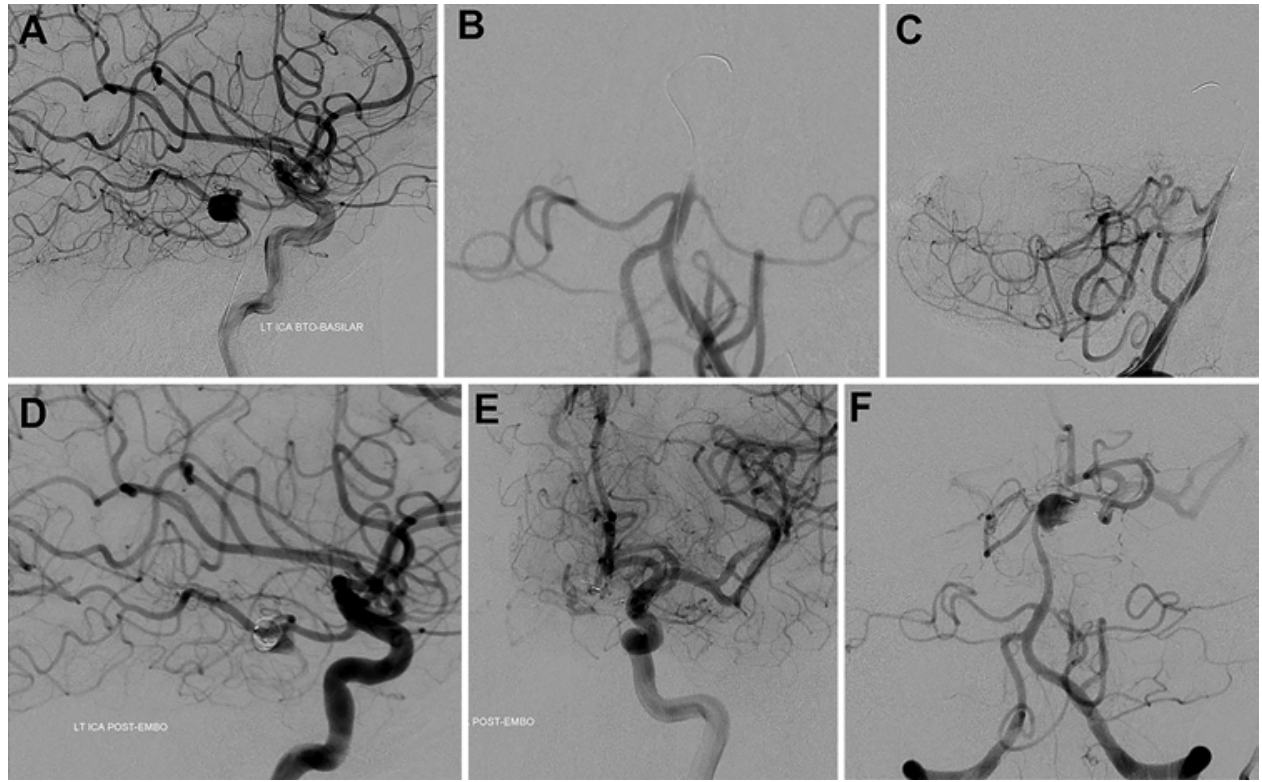

FIG. 2. Stage 1. A-C: Balloon test occlusion of the BA and sequential angiograms of the left ICA (A) and left VA (B and C) performed for evaluation of collateral reconstitution of the basilar apex. D-F: Left ICA (D and E) and left VA (F) angiograms confirming complete occlusion of the SCA component of the aneurysm after placement of a PED and MPV for mechanical occlusion of the left SCA.

and left ICA confirmed occlusion of the fusiform left SCA component of the aneurysm with collateral reconstitution of the hemispheric and vermian divisions of the left SCA through anastomoses with corresponding branches of the left anterior inferior cerebellar artery (AICA) and posterior inferior cerebellar artery (PICA) (Fig. 2D-F). The basilar component of the aneurysm was left open to supply the right SCA. The patient experienced no complications and was discharged from the neurointerventional suite in unchanged neurological condition after completion of the first stage of the intended treatment. Following the occlusion of the left SCA in stage 1, the patient was treated with a loading dose of aspirin and clopidogrel to enable subsequent reconstruction of the BAT with a PED during stage 2 .

\section{Stage 2-Treatment of the Residual Aneurysm Using a PED} From the Right SCA Into the Distal BA

Eight weeks after the deconstructive phase of embolization, the patient was brought back for the second-stage procedure. The patient was clinically stable with no new deficits since the first embolization. Despite pretreatment with aspirin and clopidogrel prior to the procedure, platelet inhibition (assessed with a P2 Y12 assay) was noted to be only partially therapeutic. Therefore, $10 \mathrm{mg}$ of abciximab (ReoPro) and $50 \mathrm{IE}$ of heparin per kilogram body weight were administered after arterial access was obtained. Diagnostic angiography confirmed occlusion of the SCA component of the aneurysm but also demonstrated an interval increase in the size of the BAT aneurysm dome compared to the stage 1 postprocedure angiogram (Fig. 3A-E).

Next a 6-Fr Envoy (supported by a 6-Fr long sheath) was placed in the left VA, and the right SCA selectively catheterized with a Phenom 27 microcatheter (Fig. 3F). The BAT was then reconstructed with a $2.5 \times 18 \mathrm{~mm}$ PED deployed distally to proximally across the aneurysm from the right SCA into the distal BA (Fig. 3G). Control angiography demonstrated no evidence of residual filling of the aneurysm from either the anterior or the posterior circulation (Fig. 3H-L). All catheters and wires were removed. Treatment with aspirin and clopidogrel was continued. This concluded the treatment strategy of the complex aneurysm, which is summarized in Fig. 4.

\section{Follow-Up and Outcome}

Postoperatively, the patient exhibited only initial mild dysmetria in her left hand, and it improved over the following 6 months. Postoperative MRI showed no diffusion abnormality (Fig. 5A). Six-month follow-up angiography confirmed successful reconstruction of the BAT with persistent occlusion of the aneurysm and maintained antegrade runoff into the right SCA (Fig. 5B-H). One-year follow-up MRI showed regression of the occluded aneurysm (Fig. 6).

\section{Discussion}

This report demonstrates the successful endovascular treatment of a complex, fusiform BAT/SCA aneurysm by staged occlusion of the left SCA followed several weeks later by PED reconstruction of the BAT. The patient recovered well from the 2-staged intervention, with improvement of her initial deficits after treatment.

This case provides new insight into the potential for extended applications of MPEDs in the management of a complex aneurysm in this location: 1) a PED was successfully used to hold an MVP device in place to occlude 

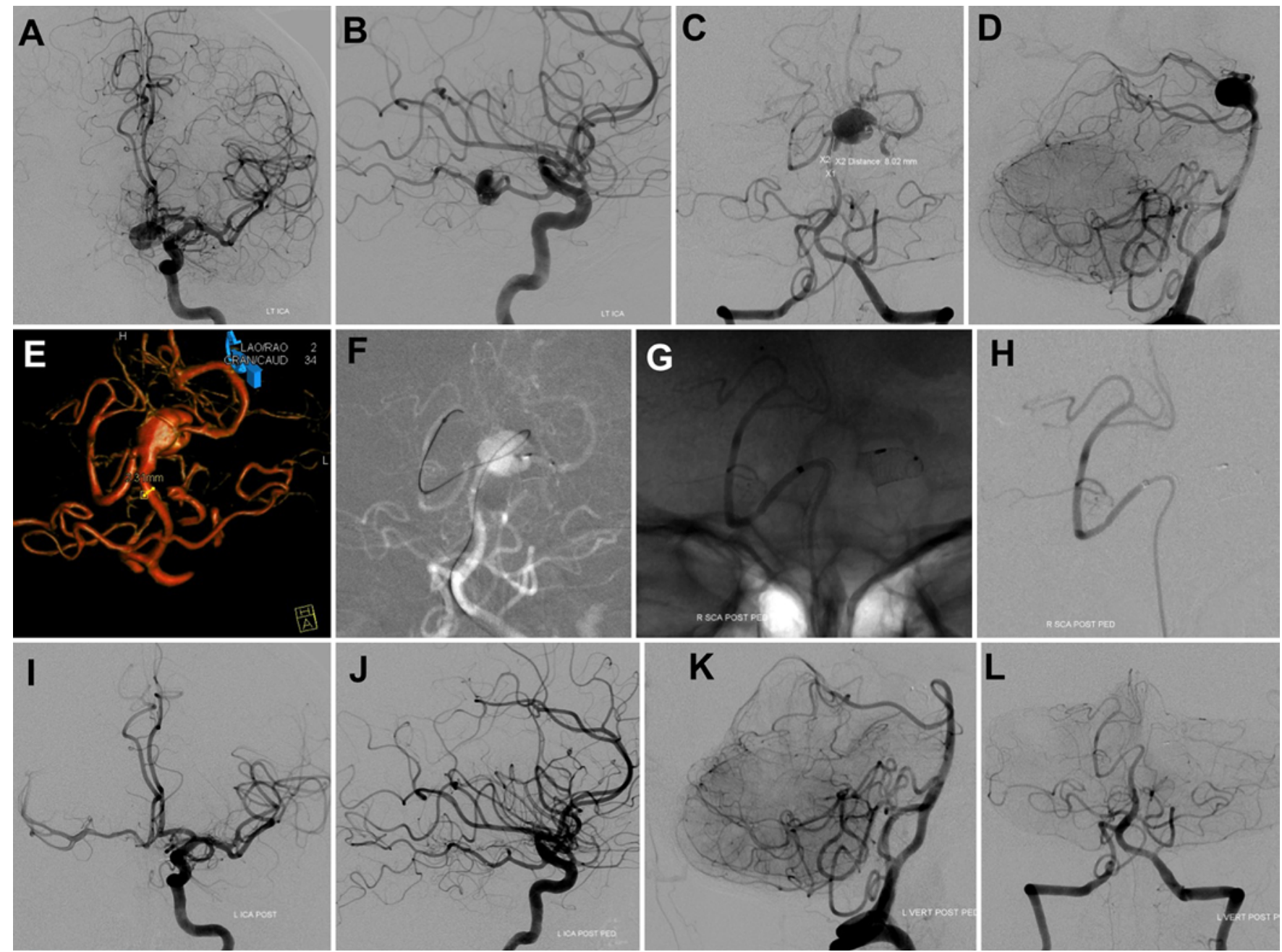

FIG. 3. Stage 2. A-D: Left ICA ( $A$ and $B)$ and left VA (C and D) angiograms obtained 8 weeks after the stage 1 procedure showing an increase of the BAT component of the aneurysm and complete occlusion of the previously treated SCA component. E-H: After $3 D$ rotational angiography of the left VA $(E)$, a microwire $(F)$ and the PED $(G)$ were placed in the right SCA $(H)$. I-L: Control angiography of the left ICA (I and J) and left VA ( $K$ and $L$ ) demonstrated no evidence of residual filling of the aneurysm from either the anterior or the posterior circulation. Figure is available in color online only.

one portion of the aneurysm (deconstructing the left SCA) prior to 2) utilizing a second PED to reconstruct the BAT and definitively occlude the remaining component of the aneurysm, exploiting the collateral arterial supply to the $\mathrm{P}_{1}$ segments (and their thalamoperforator branch vessels) through their respective PCoAs, and to the distal left SCA through corresponding hemispheric and vermian branches of the ipsilateral AICA and PICA.

Systematic studies on the treatment of posterior circulation aneurysms with flow diverters (PED or Silk flow diverter) are rare in the literature and focus mainly on unruptured aneurysms due to the necessity of adequate antiplatelet coverage. ${ }^{2,9,12}$ However, a recently published meta-analysis including 161 cases from 28 studies showed that significant numbers of aneurysms treated by flow diversion were located in the BA. ${ }^{13}$ Nevertheless, most of these aneurysms involved the basilar trunk and not, as in our case, the BAT. Besides favorable outcome in most of the cases (79\%), the aneurysm occlusion rate was also high in this meta-analysis, with $80 \%$ at 6-month follow-up with catheter angiography, which makes the flow diverter a reliable device for these aneurysms. ${ }^{13}$

The advantage of endoluminal flow diversion therapy compared to traditional stent-assisted coiling lies in the fact that direct solo-MPED treatment of the large aneurysm avoids the use of aneurysm coils and permits complete resolution of mass effect in symptomatic patients. This is especially helpful in the BAT location to avoid further mass effect on the brainstem. Although effective in altering flow within aneurysms leading to intra-aneurysmal thrombosis, coverage of small perforator branches with flow diverters such as PED is usually well tolerated, due to the diminutive size of the individual filaments composing the stent and the persistent demand for (and laminar character of) flow into covered side branches. ${ }^{4}$ Where robust collateral support provides an immediate alternative supply to the jailed vascular territory, antegrade flow across the construct is replaced by inflow from the col- 

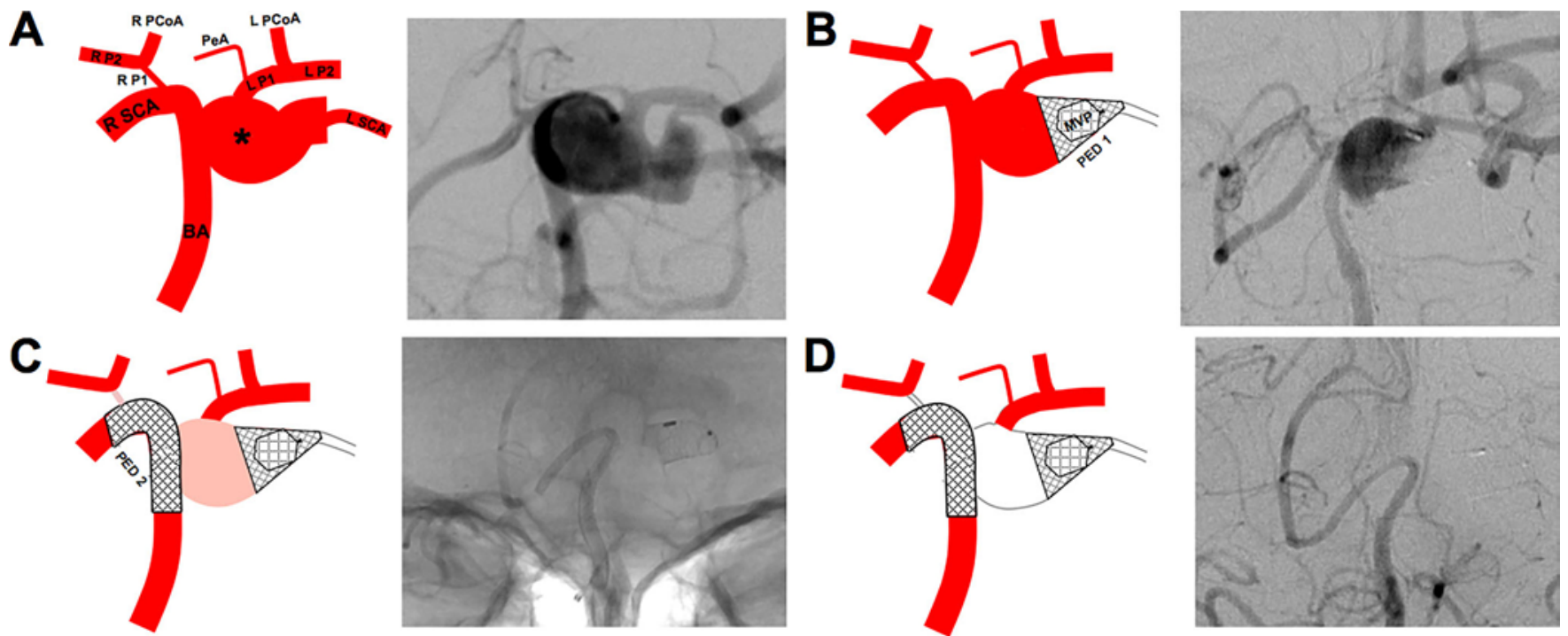

FIG. 4. Overview of the treatment strategy and device construct showing the initial anatomy before treatment (A), stage 1 (B), stage $2(\mathbf{C})$, and after treatment $(\mathrm{D})$. The asterisk $\left(^{*}\right)$ indicates the aneurysm. $\mathrm{L}=$ left; PeA = Percheron artery; $R=$ right. Figure is available in color online only.

lateral source (as in the PCoA-enabled supply of the $\mathrm{P}_{1}$ segments in this case; first demonstrated by test occlusion of the BA trunk). As in this case, the left $\mathrm{P}_{1}$ segment occludes after placement of a PED from the BAT in the right SCA, since the alternative blood supply from the left ICA through the left PCoA was more sufficient to supply the left PCA than through the PED from the BAT in this case. These considerations are not limited to the use of a single PED. In our presented case only 1 PED was needed to occlude the aneurysm, but occasionally, multiple PEDs even in more than one treatment session are needed to achieve a complete aneurysm occlusion. ${ }^{10}$
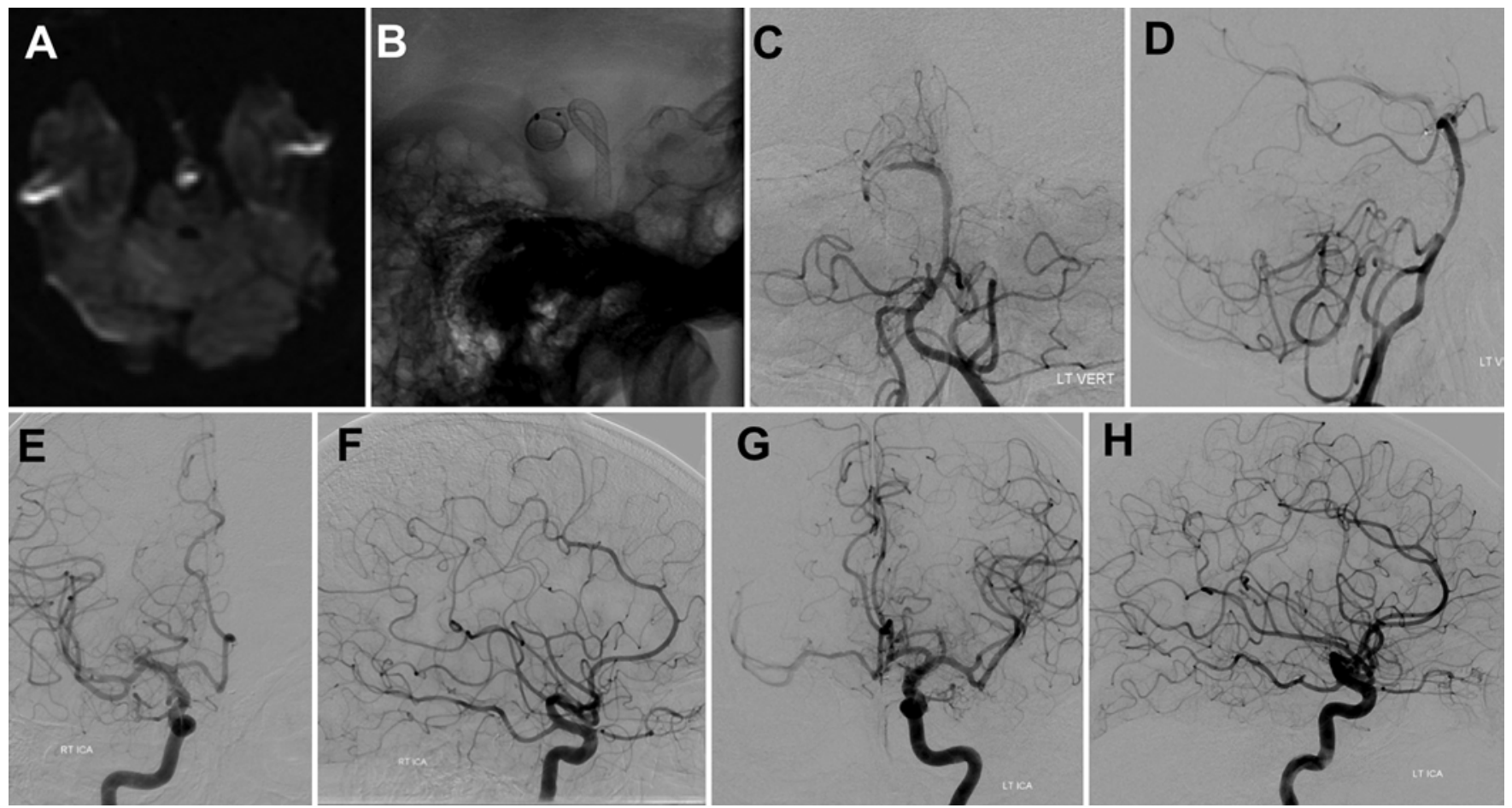

FIG. 5. Postoperative axial diffusion-weighted MR image (A) showing no infarct and 6-month follow-up angiography (B) showing the construct including PEDs and MVP in the region of the previously known complex fusiform aneurysm. Left VA injection (C and D) and both right ( $E$ and $\mathbf{F}$ ) and left ICA ( $\mathbf{G}$ and $\mathbf{H}$ ) injections showed no evidence of residual aneurysm filling. 

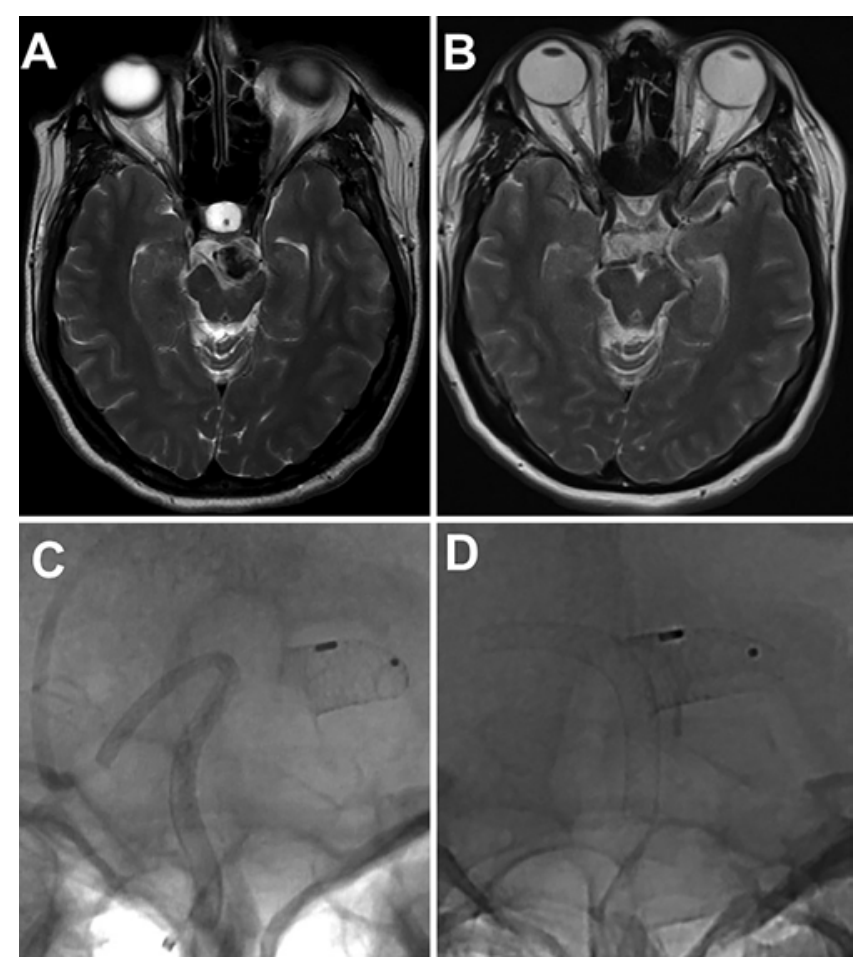

FIG. 6. A and B: Axial T2-weighted MR images obtained before any treatment $(A)$ and at approximately 6 months after stage 2 treatment $(B)$, showing regression of the aneurysm after treatment. C and D: Angiographic images obtained directly after stage 2 treatment $(C)$ and approximately 6 months later (D), showing the device construct.

In summary, this case shows that a staged treatment strategy with the initial use of a flow diverter in combination with an MVP device to deconstructively address a fusiform component of a complex BAT/SCA aneurysm was successful and that PED may be used creatively to reconstruct aberrant vascular anatomy. In this patient, the 2 -staged treatment strategy resulted in occlusion of the aneurysm and improvement of the initial clinical symptoms.

\section{References}

1. Awad AJ, Mascitelli JR, Haroun RR, De Leacy RA, Fifi JT, Mocco J: Endovascular management of fusiform aneurysms in the posterior circulation: the era of flow diversion. Neurosurg Focus 42(6):E14, 2017

2. Chalouhi N, Tjoumakaris S, Dumont AS, Gonzalez LF, Randazzo C, Starke RM, et al: Treatment of posterior circulation aneurysms with the pipeline embolization device. Neurosurgery 72:883-889, 2013

3. Coert BA, Chang SD, Do HM, Marks MP, Steinberg GK:
Surgical and endovascular management of symptomatic posterior circulation fusiform aneurysms. J Neurosurg 106:855-865, 2007

4. Fiorella D, Woo HH, Albuquerque FC, Nelson PK: Definitive reconstruction of circumferential, fusiform intracranial aneurysms with the Pipeline embolization device. Neurosurgery 62:1115-1121, 2008

5. Krisht AF, Krayenbühl N, Sercl D, Bikmaz K, Kadri PA: Results of microsurgical clipping of 50 high complexity basilar apex aneurysms. Neurosurgery 60:242-252, 2007

6. Lawton MT: Basilar apex aneurysms: surgical results and perspectives from an initial experience. Neurosurgery 50:110,2002

7. Marlin ES, Ikeda DS, Shaw A, Powers CJ, Sauvageau E: Endovascular treatment of basilar aneurysms. Neurosurg Clin N Am 25:485-495, 2014

8. Matsukawa H, Kamiyama H, Miyazaki T, Kinoshita Y, Tsuboi T, Noda K, et al: Surgical treatment of unruptured distal basilar artery aneurysm: durability and risk factors for neurological worsening. Acta Neurochir (Wien) 159:1633-1642, 2017

9. Munich SA, Tan LA, Keigher KM, Chen M, Moftakhar R, Lopes DK: The Pipeline Embolization Device for the treatment of posterior circulation fusiform aneurysms: lessons learned at a single institution. J Neurosurg 121:1077-1084, 2014

10. Shapiro M, Raz E, Becske T, Nelson PK: Building multidevice Pipeline constructs of favorable metal coverage: a practical guide. AJNR Am J Neuroradiol 35:1556-1561, 2014

11. Spetzler RF, Zabramski JM, McDougall CG, Albuquerque FC, Hills NK, Wallace RC, et al: Analysis of saccular aneurysms in the Barrow Ruptured Aneurysm Trial. J Neurosurg 128:120-125, 2018

12. Toth G, Bain M, Hussain MS, Moskowitz S, Masaryk T, Rasmussen P, et al: Posterior circulation flow diversion: a single-center experience and literature review. J Neurointerv Surg 7:574-583, 2015

13. Wang CB, Shi WW, Zhang GX, Lu HC, Ma J: Flow diverter treatment of posterior circulation aneurysms. A meta-analysis. Neuroradiology 58:391-400, 2016

\section{Disclosures}

Dr. Shapiro reports a consultant relationship with Medtronic.

\section{Author Contributions}

Conception and design: Riina, Burkhardt, Nelson. Acquisition of data: Burkhardt. Analysis and interpretation of data: all authors. Drafting the article: Burkhardt, Nelson. Critically revising the article: all authors. Reviewed submitted version of manuscript: all authors. Approved the final version of the manuscript on behalf of all authors: Burkhardt. Study supervision: Riina, Nelson.

\section{Correspondence}

Howard A. Riina: NYU Langone Hospital, New York, NY. howard.riina@nyumc.org. 